\title{
Geometrical characterization of non-Markovianity
}

\author{
Salvatore Lorenzo, ${ }^{1,2}$ Francesco Plastina, ${ }^{1,2}$ and Mauro Paternostro ${ }^{3}$ \\ ${ }^{1}$ Dipartimento di Fisica, Università della Calabria, 87036 Arcavacata di Rende, Cosenza, Italy \\ ${ }^{2}$ INFN-Gruppo Collegato di Cosenza, 87036 Arcavacata di Rende, Cosenza, Italy \\ ${ }^{3}$ Centre for Theoretical Atomic, Molecular and Optical Physics, School of Mathematics and Physics, \\ Queen's University, Belfast BT7 1NN, United Kingdom \\ (Received 20 March 2013; published 29 August 2013)
}

\begin{abstract}
We introduce a tool for the quantitative characterization of the departure from Markovianity of a given dynamical process. Our tool can be applied to a generic $N$-level system and extended straightforwardly to Gaussian continuous-variable systems. It is linked to the change of the volume of physical states that are dynamically accessible to a system and provides qualitative expectations in agreement with some of the analogous tools proposed so far. We illustrate its predictive power by tackling a few canonical examples.
\end{abstract}

DOI: $10.1103 /$ PhysRevA.88.020102

PACS number(s): 03.65.Yz, 03.65.Ta, 42.50.Lc

The interaction with an environment leads a quantum system to dissipate energy and lose its coherence. The process, however, needs not be monotonic and the system may temporarily recover some of the lost energy and/or information. This is the essence of a non-Markovian behavior, which can be characterized and quantified in different ways $[1,2]$. One possibility is to look for temporary increases of the entanglement shared by the system with an isolated ancilla, which amounts to measure the deviation from divisibility of the dynamical map describing the system's reduced evolution [3] [Rivas-Huelga-Plenio (RHP) measure]. A different approach $[4,5]$ relies on measuring the distinguishability of two optimal initial states that have evolved through the same quantum channel, looking for any nonmonotonicity [Breuer-Laine-Piilo (BLP) measure]. Further proposals are based on the decay rates entering the master equation [6], on the Fisher information flow [7], on the use of the quantum mutual information [8] or of channel capacities [9], and on spectral considerations [10]. This variety of approaches highlights the multifaceted nature of the problem of characterizing non-Markovian dynamics, which prevents the formulation of a unique tool.

In this Rapid Communication, we contribute to the quest for sharp tools able to capture the various aspects of non-Markovianity, and propose a method that qualifies non-Markovian evolutions based on the change in volume of the set of accessible states of the evolved system. As for the divisibility-based approach [3], this is a characteristic of the map itself that does not depend on the initial state(s) of the system (nor needs to be optimized over them). A quantum evolution is Markovian if it is an element of any one-parameter, continuous, completely positive semigroup: In this case the process is unidirectional and there is no recovery of energy, information, or coherence by the system. This implies that the domain's volume of the dynamical map decreases monotonically. On the contrary, we associate non-Markovianity of the dynamics to a growth of this domain's volume. By properly formalizing this intuition, we define a tool to quantify non-Markovianity, given by the sum of the (temporary) volume increases which occur during the time evolution.

In the case of a single qubit, this can be linked to the BLP measure [4], as the trace distance coincides with the Euclidean distance on the Bloch sphere and the pair of states that maximize the measure lie on the boundary of the convex subspace of physical states [11]. As a result, if the optimized trace distance decreases monotonically, so does the volume, which is, however, much easier to evaluate through the determinant of the dynamical map. We stress that ours is not yet another attempt at the quantification of the degree of non-Markovianity of a given dynamical process, but the proposal for another way to reveal physical effects (so far overlooked) of an evolution departing from Markovianity. Our proposal enjoys features of practicality and intuition of interpretation that are different from analogous, equally valid, quantifiers.

Systems with finite dimensional Hilbert spaces. Irrespective of the initial open system state, a reduced time evolution derived from the unitary dynamics of a larger system can always be described by a linear, Hermitian map [12], which is completely positive if there are no initial system-environment correlations [13]. In particular, a Markovian or memoryless behavior leads to master equations in the Lindblad form $[14,15]$, with the map obeying the semigroup composition law. Let us consider a generic completely positive trace-preserving map

$$
\phi_{t}: \hat{\rho}(0) \rightarrow \hat{\rho}(t)=\phi_{t}[\hat{\rho}(0)]
$$

Here, $\hat{\rho}$ is the quantum state of a $N$-level open system, which can be expressed through a generalized Bloch vector $\mathbf{r}$, whose components are the expectation values of the traceless, Hermitian generators of $\mathrm{SU}(N), G_{i}\left(i=1, \ldots, N^{2}-1\right)$, for which $\operatorname{Tr}\left[\hat{G}_{i} \hat{G}_{j}\right]=\delta_{i j}$. By including the identity $\hat{G}_{0}=\hat{\mathbb{I}} / \sqrt{N}$, any state can be written as

$$
\hat{\rho}=\sum_{\alpha=0}^{N^{2}-1} \operatorname{Tr}\left[\hat{\rho} \hat{G}_{\alpha}\right] \hat{G}_{\alpha} \equiv \sum_{\alpha=0}^{N^{2}-1} r_{\alpha} \hat{G}_{\alpha}
$$

with $\vec{r}=(1 / \sqrt{N}, \mathbf{r})$. A systematic construction of $\hat{G}_{\alpha}$ 's is given in Refs. [16,17] and leads to $\left\{\hat{G}_{\alpha}\right\}_{\alpha=1}^{N^{2}-1}=\left\{\hat{u}_{j k}, \hat{v}_{j k}, \hat{w}_{l}\right\} /$ $\sqrt{2}$ with

$$
\begin{aligned}
\hat{u}_{j k} & =|j\rangle\langle k|+| k\rangle\langle j|, \quad \hat{v}_{j k}=-i(|j\rangle\langle k|-| k\rangle\langle j|), \\
\hat{w}_{l} & =\sqrt{\frac{2}{l(l+1)} \sum_{j=1}^{l}(|j\rangle\langle j|-l| l+1\rangle\langle l+1|),}
\end{aligned}
$$


where $1 \leqslant j<k \leqslant N, 1 \leqslant l \leqslant N-1$, and $\{|m\rangle\}_{m=1}^{N}$ is an orthonormal basis of the open system's Hilbert space.

Writing the map (1) in this basis, one gets

$$
\vec{r}_{t}=\mathbf{F}(t) \vec{r}_{0}, \quad \text { with } \quad F_{\alpha \beta}(t)=\operatorname{Tr}\left[\hat{G}_{\alpha} \phi_{t}\left[\hat{G}_{\beta}\right]\right] .
$$

As $F_{0 \beta}(t)=\delta_{0 \beta}$, this is an affine transformation for the Bloch vector. Letting $q_{\beta}(t)=F_{\beta 0}$, we have

$$
\mathbf{F}(t)=\left(\begin{array}{cc}
1 & \mathbf{0} \\
\mathbf{q}_{t} & \mathbf{A}_{t}
\end{array}\right) \rightarrow \mathbf{r}_{t}=\mathbf{A}_{t} \mathbf{r}_{0}+\mathbf{q}_{t} / \sqrt{N} .
$$

The real matrix $\mathbf{A}_{t}$ can be decomposed as $\mathbf{A}_{t}=\mathcal{O}_{t}^{1} \mathbf{D}_{t} \mathcal{O}_{t}^{2^{T}}$, where $\mathcal{O}_{t}^{n}$ 's are orthogonal matrices and $\mathbf{D}$ is a positive semidefinite diagonal one. In what follows, we will indicate with $|M|$ the determinant of a matrix $M$. The findings above imply that $\left|\mathbf{F}_{t}\right|=\left|\mathbf{A}_{t}\right|=\left|\mathbf{D}_{t}\right|$. The action of $\mathbf{F}$ is given by a rotation (possibly composed with an inversion), a shrink of the Bloch vector, and a final rotation with possibly a translation. Its determinant gives the contraction factor of the volume of accessible states, given by the measure of the set of evolved Bloch vectors, with respect to its value at $t=0$.

The set of physical Bloch vectors for an $N$-level system is given by [18]

$$
B_{N}=\left\{\mathbf{r} \in \mathbb{R}^{N^{2}-1}:(-1)^{j} a_{j}(\mathbf{r}) \geqslant 0(j=1, \ldots, N)\right\},
$$

where $a_{j}(\mathbf{r})$ are the coefficients of the characteristic polynomial $\operatorname{det}\left(x \mathbb{I}^{N}-\hat{\rho}\right)$ with $\hat{\rho}=\frac{1}{N} \mathbb{I}^{(N)}+\sum_{i=1}^{N^{2}-1} r_{i} \hat{G}_{i}$. In spherical coordinates, the volume element of $B_{N}$ is

$$
d^{N} V=\left\|\frac{\partial\left(r_{i}\right)}{\partial\left(R, \phi_{j}\right)}\right\| d R d \phi_{1} d \phi_{2} \cdots d \phi_{n-1} .
$$

Any positive trace-preserving map described by Eq. (4) induces the change $d^{N} V(t)=\left\|\mathbf{A}_{t}\right\| d^{N} V(0)$. Therefore $\left\|\mathbf{A}_{t}\right\|$ describes the change in volume of the set of states accessible through the evolution of the reduced state. In particular, $\left\|\mathbf{A}_{t}\right\|$ decreases monotonically for any positive, linear, and trace-preserving map [19], and so it does for any element of a completely positive continuous one-parameter semigroup. Indeed, if $\hat{\phi}_{t}=\exp [t \hat{\mathcal{L}}]$ with

$$
\hat{\mathcal{L}} \hat{\rho}=i[\hat{\rho}, \hat{H}]+\sum_{\alpha, \beta} \gamma_{\alpha, \beta}\left(\hat{C}_{\alpha} \hat{\rho} \hat{C}_{\beta}^{\dagger}-\frac{1}{2}\left\{\hat{C}_{\beta}^{\dagger} \hat{C}_{\alpha}, \hat{\rho}\right\}\right),
$$

$\gamma_{\alpha, \beta} \geqslant 0$, and $\hat{H}=\hat{H}^{\dagger}$, then $\left|\mathbf{A}_{t}\right|$ reduces to a constant.

Generators of the form in Eq. (7), but with explicitly time-dependent coefficients or jump operators lead to timedependent Markovian processes. Indeed, although not being part of a dynamical semigroup, the corresponding dynamical map $\hat{\phi}_{t+\tau, t}=\exp \left[T_{d} \int_{t}^{t+\tau} \hat{\mathcal{L}} d t\right]\left(T_{d}\right.$ is the time-ordering operator) is divisible and can always be written as a composition of two CPT maps

$$
\hat{\phi}_{t+\tau, 0}=\hat{\phi}_{t+\tau, t} \hat{\phi}_{t, 0} \quad(\forall \tau, t \geqslant 0) .
$$

As a consequence, in this case too the determinant decreases monotonically.

These considerations lead us to define a way to quantify the non-Markovianity of a quantum evolution through the variation of the volume of accessible states

$$
\mathcal{N}_{V}=\frac{1}{V(0)} \int_{\partial_{t} V(t)>0} \partial_{t} V(t)=\int_{\partial_{t}\left\|\mathbf{F}_{t}\right\|>0} \partial_{t}\left\|\mathbf{F}_{t}\right\| .
$$

(a)
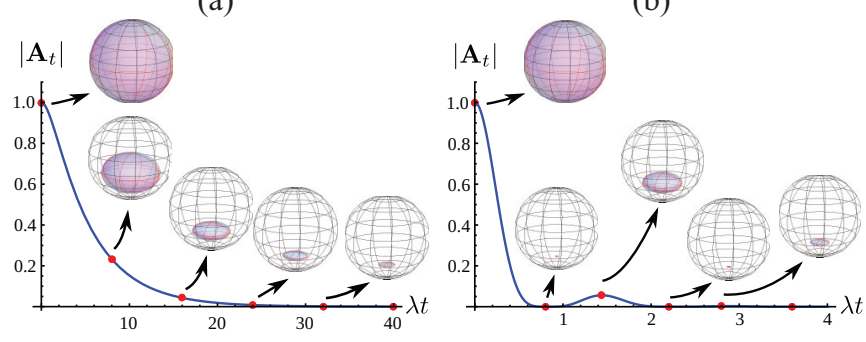

FIG. 1. (Color online) Time evolution of the determinant of the map for a generic Markovian (a) and non-Markovian dynamics (b). Quantitatively, the curves displayed in the figure correspond to the spontaneous emission of a two-level system in a resonant leaky cavity (cf. Example 1) with the Lorentzian spectral density given in Eq. (13). (a) shows the (Markovian) case with $\gamma_{0} / \lambda=0.1$ (bad cavity limit), while (b) shows a (non-Markovian) evolution in the good cavity limit with $\gamma_{0} / \lambda=10$. We also picture the set of accessible states, whose volume changes in time according to the behavior of the determinant.

The intuitive meaning of this definition is illustrated in Fig. 1, where the time evolution of the determinant is explicitly shown for the case of a two-level atom spontaneously decaying in a structured environment (cf. Example 1 for details). The monotonic decay of the volume characterizing a Markovian evolution is contrasted with a non-Markovian behavior where the volume of accessible states returns to be nonzero after having vanished.

Besides the geometric interpretation, the proposed measure can be linked to the change of the classical information encoded in the states of a system. Suppose that a set of quantum states is given with elements characterized by an arbitrary distribution of Bloch vectors within $B_{N}$ and described by a probability density $p(\mathbf{r})$. The corresponding differential entropy is $h[p(\mathbf{r})]=-\int_{B_{N}} p(\mathbf{r}) \log _{2} p(\mathbf{r}) d V_{N}$. If the states are evolved through $\phi_{t}$, after a time $\tilde{t}$ the probability density function is rescaled as $p^{\prime}\left(\mathbf{r}_{\tilde{t}}\right)=p\left(\mathbf{r}_{\tilde{t}}\right) /\left\|\mathbf{A}_{\tilde{t}}\right\|$ and the entropy changes as $h\left[p^{\prime}\left(\mathbf{r}_{\tilde{t}}\right)\right]-h\left[p\left(\mathbf{r}_{0}\right)\right]=\log _{2}\left\|\mathbf{A}_{\tilde{t}}\right\|$. A volume contraction thus implies a loss of information.

The BLP measure also enjoys an information theoretical interpretation, but a comparison between the two quantifiers is difficult. Indeed, although it is known that the optimal states that enter the measure proposed in Ref. [4] lie on the boundary of the volume accessible throughout the dynamics [11], this does not necessarily imply a connection with the measure of such volume. On the other hand, the difference between $\mathcal{N}_{V}$ and the RHP measure is simpler to describe: As the determinant is contractive under composition of positive maps, it follows that it does not increase whenever the intermediate map $\phi_{t+\tau, t}$ in Eq. (8) is positive. The entanglement-based measure of Ref. [3], on the other hand, is zero in the more restrictive condition of this map being completely positive. Explicitly, if $\phi_{t+\tau, t}$ is completely positive $(\forall t, \tau)$, then the dynamics is Markovian, according to both the RHP and criterion and to ours. On the other hand, if $\phi_{t+\tau, t}$ is positive but not completely positive, the map is nondivisible, and thus non-Markovian in the sense of RHP; nonetheless, it is Markovian according to our volume quantifier, $\mathcal{N}_{V}=0$.

From a practical viewpoint, the experimental evaluation of $\mathcal{N}_{V}$ passes through the determination of the volume of the set 
(a)

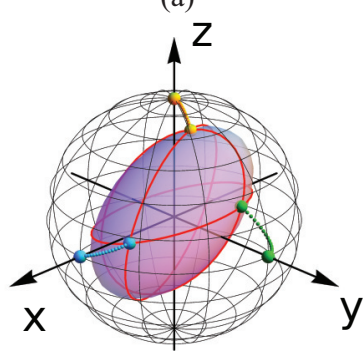

(b)

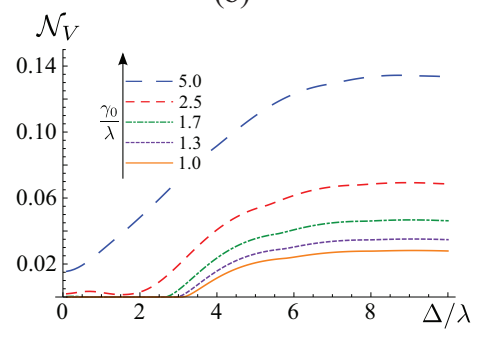

FIG. 2. (Color online) (a) Example of an ellipsoid representing the image of a random map for a qubit. The canonical basis of $\mathbb{R}^{3}$ is mapped onto the corresponding colored circles highlighted on the ellipsoid, from which the volume can be obtained. (b) $\mathcal{N}_{V}$ against $\Delta$ for the spontaneous-emission dynamics into a reservoir with Lorentzian spectral density (cf. Example 1).

of evolved states found from the contraction of $B_{N}$. This is an ellipsoid for the case of a qubit. As the number of states that any realistic experimental implementation can sample is finite, it would be a precious piece of information to know which are the best initial states to use in order to determine the set of physically accessible states at given time $t$ and its volume. For this purpose, let us consider $N$ initial Bloch vectors, evolved up to time $t$ and arranged as the columns of a matrix $\mathbf{P}_{t}$. From Eq. (5), we find $\mathbf{P}_{t}=\mathbf{A}_{t} \mathbf{P}_{0}+\mathbf{Q}_{t}$, where the columns of $\mathbf{Q}_{t}$ are given by $\mathbf{q}_{t}$ 's (which is the evolved state for a maximally mixed initial condition). It then follows that $\mid\left(\mathbf{P}_{t}-\mathbf{Q}_{t}\right)$ $\left(\mathbf{P}_{t}-\mathbf{Q}_{t}\right)^{T} \mid=\left(\left|\mathbf{A}_{t}\right|\left|\mathbf{P}_{0}\right|\right)^{2}$. Thus, if we choose as initial Bloch vectors the elements of any orthogonal basis in $\mathbb{R}^{N^{2}-1}$ plus the null vector corresponding to the maximally mixed state, then their time evolutions (arranged to form the matrix $\mathbf{P}_{t}-\mathbf{Q}_{t}$ ) gives the determinant of the map, from which the measure $\mathcal{N}_{V}$ easily follows. Therefore, the geometric measure of non-Markovianity in Eq. (9) can be revealed experimentally by performing a state tomography at different times for $N^{2}-1$ initial orthogonal states. This will be sufficient to evaluate the change in volume of the accessible states without the need for prior knowledge about the environment or the coupling. This is illustrated in Fig. 2(a) for a qubit: The vectors associated with the canonical basis of $\mathbb{R}^{3}$ are mapped onto the corresponding points on surface of the ellipsoid that comprises all the possible accessible states of the evolution. Incidentally, the problem of finding minimum volume covering ellipsoids has been studied extensively and efficient algorithms exist for the computation of such volume given $n$ points in $\mathbb{R}^{N}$ (a noticeable example being in Ref. [20]).

Systems with infinite dimensional Hilbert space. We can extend our idea to the less intuitive context of continuousvariable systems, in which the Hilbert space is infinite dimensional, provided we restrict to Gaussian state and Gaussian-preserving processes.

We consider a system made of $n$ bosonic modes, each described by the annihilation (creation) operators $\hat{a}_{k}\left(\hat{a}_{k}^{\dagger}\right), k=$ $1, \ldots, n$ [the corresponding quadratures are $\hat{q}_{k}=1 / \sqrt{2}\left(\hat{a}_{k}+\right.$ $\left.\hat{a}_{k}^{\dagger}\right)$ and $\left.\hat{p}_{k}=-i / \sqrt{2}\left(\hat{a}_{k}-\hat{a}_{k}^{\dagger}\right)\right]$. Defining the vector of operators $\hat{\mathbf{R}}=\left(\hat{q}_{1}, \hat{p}_{1}, \ldots, \hat{q}_{n}, \hat{p}_{n}\right)^{T}$, the commutation relations can be written as $\left[\hat{R}_{k}, \hat{R}_{l}\right]=i \Omega_{k l}$, where $\Omega_{k l}$ are elements of the symplectic matrix $\boldsymbol{\Omega}=\oplus_{k=1}^{n} \boldsymbol{\omega}$ with $\boldsymbol{\omega}=\left(\begin{array}{cc}0 & 1 \\ -1 & 0\end{array}\right)$. A Gaussian state is characterized by the first two statistical moments. The first, $\langle\mathbf{R}\rangle$, can be adjusted to be null by local unitary operations; the second is given by the covariance matrix $\sigma, \sigma_{k l}=\frac{1}{2}\left\langle\left\{\hat{R}_{k}, \hat{R}_{l}\right\}\right\rangle-\left\langle\hat{R}_{k}\right\rangle\left\langle\hat{R}_{l}\right\rangle$. It can be shown that any dynamics resulting from the reduction of a symplectic evolution on a larger Hilbert space can be described by

$$
\sigma_{0} \rightarrow \sigma_{t}=\xi_{t}^{T} \sigma_{0} \xi_{t}+\psi_{t},
$$

where $\xi_{t}$ and $\psi_{t}$ are $2 n \times 2 n$ real matrices fulfilling the relation $\psi_{t}+i \boldsymbol{\Omega}-i \xi_{t}^{T} \boldsymbol{\Omega} \xi_{t} \geqslant 0$. Vice versa, any evolution of this kind may be interpreted as the reduction of a larger symplectic evolution [21]. In full analogy with what we did in Eq. (4), by choosing a basis $\left\{G_{j}\right\}$ for the space of $2 n \times 2 n$ matrices, we can write such map as the $\mathbb{R}^{4 n^{2}}$ affine transformation

$$
\mathbf{s}_{0} \rightarrow \mathbf{s}_{t}=\mathbf{X}_{t} \mathbf{s}_{0}+\mathbf{Y}_{t},
$$

with $s_{k}(\tau)=\operatorname{Tr}\left[\sigma_{\tau} G_{k}\right], X_{k j}(\tau)=\operatorname{Tr}\left[\xi_{\tau}^{T} G_{k} \xi_{\tau} G_{j}\right]$, and $Y_{j}(\tau)=$ $\operatorname{Tr}\left[\psi_{\tau} G_{j}\right]$.

We then define a measure of non-Markovianity in a way fully analogous to what has been done above for the case of a discrete-variable system, i.e., as in Eq. (9) with the replacement $\mathbf{F}_{t} \rightarrow \mathbf{X}_{t}$.

If the evolution is unitary, the associated transformation has a constant determinant. For a single mode in a generic noisy Markovian channel, we have [22]

$$
\sigma_{t}=e^{-\Gamma t} \sigma_{0}+\left(1-e^{-\Gamma t}\right) \sigma_{\infty}
$$

which gives $\left|\mathbf{X}_{t}\right|=e^{-4 \Gamma t}$. Therefore, every increase in $\left|\mathbf{X}_{t}\right|$ signals non-Markovianity.

Having introduced our formalism, we now illustrate our proposal with the aid of a few significant examples.

Example 1: Spontaneous emission into a leaky cavity. Consider a single two-level atom with transition frequency $\omega_{0}$ interacting with a vacuum electromagnetic field having a Lorentzian spectral density (mimicking a leaky cavity) [5,23]. Taking

$$
J(\omega)=\frac{1}{2 \pi} \frac{\gamma_{0} \lambda^{2}}{\left(\omega_{0}-\Delta-\omega\right)^{2}+\lambda^{2}},
$$

where $\Delta$ is the detuning between the atomic and the cavity frequency, the atomic state at time $t$ reads

$$
\hat{\rho}_{A}(t)=\left(\begin{array}{cc}
|\Gamma(t)|^{2} \rho_{0}^{++} & \Gamma(t) \rho_{0}^{+-} \\
\Gamma(t)^{*} \rho_{0}^{-+} & \left(1-|\Gamma(t)|^{2}\right) \rho_{0}^{++}+\rho_{0}^{--}
\end{array}\right),
$$

with $\Omega_{ \pm}=\Delta-i \lambda \pm \sqrt{(\Delta-i \lambda)^{2}+2 \gamma_{0} \lambda}$ and

$$
\Gamma(t)=\frac{e^{-\frac{i t \Omega_{-}}{2}} \Omega_{+}-e^{-\frac{i t \Omega_{+}}{2}} \Omega_{-}}{2\left(\Omega_{+}-\Delta+i \lambda\right)} .
$$

The evolution of the Bloch vector is ruled by

$$
\mathbf{A}_{t}=\left(\begin{array}{ccc}
\operatorname{Re} \Gamma(t) & \operatorname{Im} \Gamma(t) & 0 \\
-\operatorname{Im} \Gamma(t) & \operatorname{Re} \Gamma(t) & 0 \\
0 & 0 & |\Gamma(t)|^{2}
\end{array}\right)
$$

whose determinant, $\left|\mathbf{A}_{t}\right|=|\Gamma(t)|^{4}$, is shown in Fig. 1 against the dimensionless time $\lambda t$. The corresponding nonMarkovianity measure $\mathcal{N}_{V}$ is reported in Fig. 2(b), from which it is clear that a strongly non-Markovian behavior is found 
for a resonant coupling in the so-called good-cavity limit $\gamma_{0} \gg \lambda$. A similar result is obtained with the RHP measure, [3] which is given by the integral of $(1 / 2) \operatorname{Re}\left[\partial_{t} \ln \Gamma(t)\right]$. This is in agreement with BLP as well, which turns out to depend on $|\Gamma(t)|[5]$.

Example 2: Pure dephasing. Let us consider a qubit undergoing a purely dephasing dynamics, expressed in terms of a decoherence factor $v(t)$ as

$$
\hat{\phi}_{t}^{(d)}[\rho(0)]=\left(\begin{array}{cc}
\rho^{++} & v(t) \rho^{+-} \\
v(t) \rho^{-+} & \rho^{--}
\end{array}\right) .
$$

In this case, the BLP and RHP measures coincide [24,25]. In fact, the optimized trace distance gives $D\left[\rho_{1}^{\mathrm{opt}}, \rho_{2}^{\mathrm{opt}}\right]=$ $|v(t)|$. In turn, $|v(t)|$ is exactly the value of the concurrence between a system qubit and ancilla, initially prepared in a maximally entangled state and undergoing a unilateral dephasing mechanism. As for our proposal, the evolution of the Bloch vector is determined by the matrix

$$
\mathbf{A}_{t}=\left(\begin{array}{ccc}
\operatorname{Re} v(t) & \operatorname{Im} v(t) & 0 \\
-\operatorname{Im} v(t) & \operatorname{Re} v(t) & 0 \\
0 & 0 & 1
\end{array}\right)
$$

for which $\left\|\mathbf{A}_{t}\right\|=|v(t)|^{2} . \mathcal{N}_{V}$ thus gives the same behavior predicted by the other two quantifiers.

Example 3: Harmonic oscillators. We consider a single quantum harmonic oscillator that interacts with a reservoir of $N_{m}$ modes as

$$
\hat{H}=\omega_{0}\left(\hat{q}_{0}^{2}+\hat{p}_{0}^{2}\right)+\omega \sum_{j=1}^{N_{m}}\left(\hat{q}_{j}^{2}+\hat{p}_{j}^{2}\right)+\kappa \hat{q}_{0} \sum_{j=1}^{N_{m}} \hat{q}_{j} .
$$

When expressed in terms of bosonic annihilation and creation operators, the interaction term in Eq. (19) includes both rotating and counter-rotating terms. The latter do not preserve energy and induce squeezing in the system, in turn resulting in an effective growth of the determinant of the map above its initial value. In what follows, we will work assuming that the total energy of the system is fixed, and thus invoke a rotatingwave approximation that allows us to neglect counter-rotating terms and reduce the system-environment coupling to the generator of beamsplitterlike transformations $\kappa \hat{q}_{0} \sum_{j=1}^{N_{m}} \hat{q}_{j} \rightarrow$ $(\kappa / 2) \hat{a}_{0} \sum_{j=1}^{N_{m}} \hat{a}_{j}^{\dagger}+$ H.c. The quadratic nature of this model allows us to restrict our analysis to input Gaussian states and thus use the formalism of covariance matrices and symplectic transformations to describe the evolution [22]. Following Ref. [26], the dynamics induced by the chosen model transforms the input covariance matrix $\sigma_{0}$ of the $N_{m}+1$-mode system as $\sigma_{t}=\mathcal{T} \sigma_{0} \mathcal{T}^{T}$ with the symplectic transformation $\mathcal{T}=\mathcal{T}_{B_{\text {coll }}} \mathcal{T}_{R_{\text {coll }}} \mathcal{T}_{B_{\text {coll }}}^{T}$ composed of a series of pairwise beamsplitters $\left(\mathcal{T}_{B_{\text {coll }}}\right)$ and the tensor product of singlemode rotations in phase space $\mathcal{T}_{R_{\text {coll }}}$. We refer to Ref. [26] for the form of such transformations.

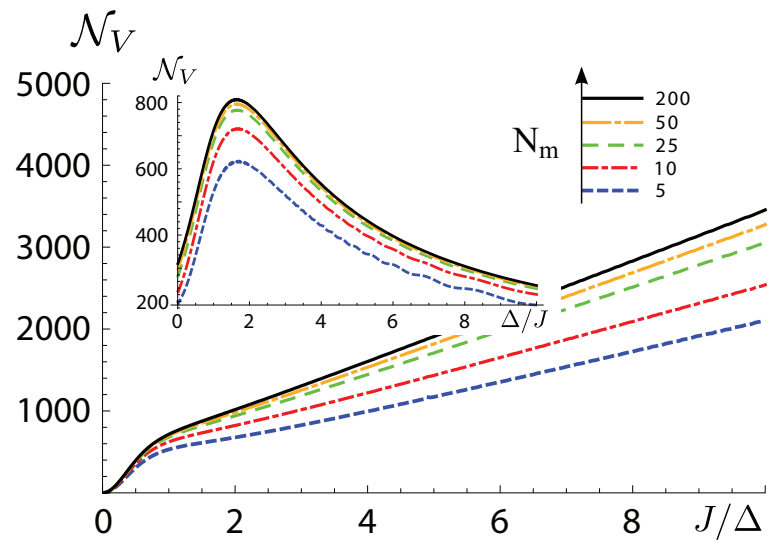

FIG. 3. (Color online) Non-Markovianity measure $\mathcal{N}_{V}$ as a function of $J / \Delta$ (with the coefficient $J$ defined through $\kappa=$ $\sqrt{\Delta^{2}+J^{2} / N_{m}}$ ), and at various values of $N_{m}$. The inset shows $\mathcal{N}_{V}$ against the detuning $\Delta=\omega-\omega_{0}$. The measure is calculated integrating over the time window $[0,4000 / \kappa]$.

Here, it is enough to mention that, by choosing a factorized initial state for the system and reservoir, the evolution of the central oscillator can be found by tracing over the degrees of freedom of the $N_{m}$ environmental modes and can be cast into the form of Eq. (10) with

$$
X_{t}=\left(\begin{array}{cc}
\sqrt{\mathcal{T}_{11}{ }^{2}+\mathcal{T}_{12}{ }^{2}} & \frac{\mathcal{T}_{11} \mathcal{T}_{21}+\mathcal{T}_{12} \mathcal{T}_{22}}{\sqrt{\mathcal{T}_{11}{ }^{2}+\mathcal{T}_{12}{ }^{2}}} \\
0 & -\frac{\sqrt{\left(\mathcal{T}_{12} \mathcal{T}_{21}-\mathcal{T}_{11} \mathcal{T}_{22}\right)^{2}}}{\sqrt{\mathcal{T}_{11}{ }^{2}+\mathcal{T}_{12}{ }^{2}}}
\end{array}\right)
$$

and $\mathcal{T}_{i j}$ 's the entries of the symplectic matrix associated with $\mathcal{T}$. We finally have $\left|\mathbf{X}_{t}\right|=\left(\mathcal{T}_{12} \mathcal{T}_{21}-\mathcal{T}_{11} \mathcal{T}_{22}\right)^{4}$, whose behavior within a fixed time interval of the evolution is shown in Fig. 3, evidencing non-Markovianity due to the backflow of excitations to the central mode.

Conclusions. We have proposed a geometrically motivated quantifier of non-Markovianity $\mathcal{N}_{V}$ explicitly linked to the variations of the volume of the physical states dynamically accessible by a given open system. From an information theoretical perspective, such a measure is linked to the loss or regain of classical information over the evolving system. We have shown how $\mathcal{N}_{V}$ can be estimated through only a polynomial number of measures, and that it enjoys a straightforward extension to the Gaussian continuous-variable scenario. Finally, we have illustrated our proposal through three examples that emphasize its appealing aspects of practicality and intuitive nature.

Acknowledgments. We thank T. J. G. Apollaro, J. Goold, and A. Rivas for relevant discussions on the topics of this article. S.L. thanks the Centre for Theoretical Atomic, Molecular and Optical Physics for hospitality during the early stages of this work. M.P. thanks the EPSRC for financial support through a Career Acceleration Fellowship and a grant awarded under the "New Directions for Research Leaders" initiative (EP/G004579/1).
[1] M. M. Wolf, J. Eisert, T. S. Cubitt, and J. I. Cirac, Phys. Rev. Lett. 101, 150402 (2008).

[2] D. Chruscinski and A. Kossakowski, Phys. Rev. Lett. 104, 070406 (2010)
[3] A. Rivas, S. F. Huelga, and M. B. Plenio, Phys. Rev. Lett. 105, 050403 (2010).

[4] H.-P. Breuer, E.-M. Laine, and J. Piilo, Phys. Rev. Lett. 103, 210401 (2009). 
[5] E.-M. Laine, J. Piilo, and H.-P. Breuer, Phys. Rev. A 81, 062115 (2010).

[6] E. Andersson, J. D. Cresser, and M. J. W. Hall, arXiv:1009.0845.

[7] X.-M. Lu, X. Wang, and C. P. Sun, Phys. Rev. A 82, 042103 (2010).

[8] S. Luo, S. Fu, and H. Song, Phys. Rev. A 86, 044101 (2012).

[9] B. Bylicka, D. Chruściński, and S. Maniscalco, arXiv:1301.2585.

[10] W. M. Zhang, P. Y. Lo, H. N. Xiong, M. W.-Y. Tu, and F. Nori, Phys. Rev. Lett. 109, 170402 (2012); A. Sindona et al., arXiv:1211.1398.

[11] S. Wißmann, A. Karlsson, E.-M. Laine, J. Piilo, and H.-P. Breuer, Phys. Rev. A 86, 062108 (2012).

[12] A. Shabani and D. A. Lidar, Phys. Rev. A 80, 012309 (2009).

[13] T. F. Jordan, A. Shaji, and E. C. G. Sudarshan, Phys. Rev. A 70, 052110 (2004).

[14] G. Lindbald, Commun. Math. Phys. 48, 119 (1976).

[15] V. Gorini, A. Kossakowski, and E. C. G. Sudarshan, J. Math. Phys. 17, 821 (1976)
[16] G. Mahler and V. A. Weberruss, Quantum Networks (Springer, Berlin, 1995).

[17] R. Alicki and K. Lendi, Quantum Dynamical Semigroups and Application (Springer, Berlin, 1987).

[18] G. Kimura, J. Phys. Soc. Jpn. 72 (2003) Supplement C 185.

[19] M. M. Wolf and J. I. Cirac, Commun. Math. Phys. 279, 147168 (2008).

[20] M. J. Todd and E. A. Yıldırım, Discrete Appl. Math. 155, 13 (2007).

[21] A. Serafini, J. Eisert, and M. M. Wolf, Phys. Rev. A 71, 012320 (2005).

[22] A. Ferraro, S. Olivares, and M. G. A. Paris, Gaussian States in Quantum Information (Bibliopolis, Napoli, 2005).

[23] F. Francica, S. Maniscalco, J. Piilo, F. Plastina, and K.-A. Suominen, Phys. Rev. A 79, 032310 (2009).

[24] H.-S. Zeng, N. Tang, Y.-P. Zheng, and G.-Y. Wang, Phys. Rev. A 84, 032118 (2011).

[25] P. Haikka, J. Goold, S. McEndoo, F. Plastina, and S. Maniscalco, Phys. Rev. A 85, 060101 (2012).

[26] M. Paternostro, M. S. Kim, E. Park, and J. Lee, Phys. Rev. A 72, 052307 (2005). 